\title{
Idée de l'homme et apprentissage des concepts géographiques à l'école
}

\begin{abstract}
Ce texte reprend les idées principales de l'introduction du livre consacré à la géographie à l'école que trois membres de la Commission "Evaluation et Didactique de la Géographie" (C. Davaud, M. Solari et P. Varcher) vont publier très prochainement. Cette commission a travaillé dans le cadre du Centre de Recherches Psycho-pédagogiques (CRPP) du Cycle d'Orientation de Genève.
\end{abstract}

Si l'école reste avant tout le lieu d'acquisition de savoirs, les connaissances et les compétences que les élèves vont y acquérir nont de sens que si elles contribuent à développer ce qui fait la particularité de l'humain; or, ce développement suppose qu'on fasse émerger une "conscience planétaire», au sens où l'entend Edgar Morin, et qu'on accepte le préalable du refus de la violence:

- L'émergence de la "conscience planétaire», c'est la prise de conscience, par tous, de la communauté de destin de tous les hommes car, comme l'a écrit Albert Jacquard, "voici le temps du monde fini» (JACQUARD 1991). Edgar Morin précise: «A l'ancien substrat bioanthropologique qui constitue l'unité de l'espèce humaine s'ajoute désormais un tissu communicationnel, civilisationnel, culturel, économique, technologique, intellectuel, idéologique. L'espèce humaine nous apparaît désormais comme humanité. (...) Bien qu'il y ait désormais une communauté de destin, il n'y a pas encore conscience commune de cette Schicksalsgemeinschaft» (MORIN/KERN 1993, p. 42). Contribuer à l'émergence de cette conscience planétaire doit devenir une des finalités de l'école, tout en étant conscient que cette émergence ne saurait s'effectuer sans "préserver et sauvegarder nos diversités culturelles et naturelles» ainsi que "nos acquis civilisationnels»; elle ne «pourrait être atteinte qu'en révolutionnant partout les relations entre humains, depuis les relations de soi à soi, de soi à autrui et entre proches, jusqu'aux relations entre nations et Etats et aux relations entre les hommes et la technobureaucratie, entre les hommes et la société, entre les hommes et la connaissance, entre les hommes et la nature» (MORIN/KERN 1993, p. 117).

- Révolutionner toutes ces relations présuppose la recherche d'une coexistence basée sur le préalable du refus de la violence, un préalable qu'il n'est pas besoin de justifier «car il n'y a rien en amont du refus de la violence, rien qui lui soit fondateur, que ce refus de la violence luimême en tant qu'il est expression irréductible d'humanité» (MEIRIEU 1993, p. 103). Certes, «l'évocation d'une humanité où les conflits seraient résolus sans recours à la violence se heurte, au mieux, au sourire sceptique des "réalistes": il y a toujours eu des guerres, il y en aura toujours, la violence est dans la nature humaine» (JACQUARD 1990, p. 168). Mais, comme Jacquard, nous pensons que "cette affirmation n'est nullement prouvée. Le serait-elle, l'acceptation d'une fatalité n'en serait pas justifiée. (...) Le choix est clair: ou nous laissons cette violence, éventuellement «naturelle», aujourd'hui multipliée par les moyens techniques mis à son service, continuer à se répandre dans nos pensées et nos actes, et l'humanité ne survivra pas plus de quelques dizaines d'années; ou nous saurons instaurer des rapports entre individus et entre nations basés sur un modèle enfin raisonnable, et la survie peut être obtenue. (...) Désormais la solidarité la plus nécessaire est celle de l'ensemble des habitants de la terre.»

Dans le cadre d'un projet éducatif tendant au développement de ce qui fait la particularité de l'humain, la géographie a-t-elle un rôle à jouer? Assurément et plus que jamais! «La société devenant de plus en plus diverse et plus sensible aux conflits internes, il faut être particulièrement attentif au rôle de l'école dans la promotion de la diversité. L'objectif ultime doit être de mettre en valeur la richesse de l'humanité tout en faisant mieux sentir la nécessité de la cohésion sociale» (UNESCO 1994). Certes, cette ambition doit traverser toute l'école, être présente dans toutes les disciplines, mais les sciences humaines ont un rôle privilégié à remplir et s'avèrent de plus en plus indispensables dans une école qui doit préparer les citoyens du XXe siècle à affronter les grands défis sociaux qui s'annoncent: les migrations internationales, le déséquilibre nord-sud, la création de grands ensembles politico-économiques supranationaux, la montée des nationalismes, les conséquences sociales de l'amélioration de la productivité dans tous les secteurs de l'économie et des besoins réduits en main-d'œuvre qu'elle entraîne... L'école se doit de permettre aux jeunes de se construire des aptitudes, des compétences et des savoirs suffisants pour aborder la complexité de tels problèmes et donc de réserver un domaine d'études obligatoire à cela. Il nous paraît important d'affirmer que la complexité des problèmes évoqués ci-dessus ne peut être abordée

Pierre Varcher, 2, rue Beau-Site, 1203 Genève 
qu'en faisant appel aux différentes composantes des sciences humaines et sociales, dont la géographie. Cette complexité exige une approche à la fois diachronique et synchronique et l'approche historique en sciences humaines est dès lors incontournable; mais l'histoire ne peut prétendre fournir des aptitudes et des compétences suffisantes pour une approche systémique des phénomènes sociaux. L'apport spécifique d'autres disciplines, dont la géographie, s'avère alors indispensable. Si nous pensons que la géographie est, à l'école, la discipline la mieux à même de promouvoir un apprentissage de connaissances et de compétences permettant une prise synchronique sur le "réel», et qu'elle peut être plus efficace pour des enfants et des adolescents que la sociologie par exemple, c'est parce que son ancrage sur l'espace donne aux apprenants une prise sur quelque chose de relativement concret; mais ancrer des problématiques sur l'espace ne signifie pas se limiter à l'étude de l'espace physique. L'objet de la géographie à l'école ne doit plus être l'espace mais les relations que les hommes nouent avec l'espace. Mais une telle géographie impose la construction et l'utilisation de modèles: «La "chose» géographique immédiatement visible, un paysage par exemple, peut être naturellement décrit au moyen d'une langue naturelle quelconque mais cela ne dépasse pas, alors, l'appréhension commune. Si l'objet du géographe est «un système de relations à l'espace», ce système doit être construit. En effet, si les relations sont déchiffrables, elles ne sont pas, au sens strict du terme, visibles. En revanche, elles sont visualisables mais, pour les rendre telles, il faut élaborer un appareil conceptuel» (RAFFESTIN/TURCO 1991). C'est avec cet appareil conceptuel qu'un élève doit apprendre à se familiariser: penser les relations de l'homme avec l'espace, c'est apprendre à organiser ses perceptions et ses connaissances, c'est apprendre à manipuler des concepts et à se poser les questions spécifiques que le géographe se pose. Ainsi, par exemple, le concept «échelle» ne consiste pas à savoir faire les opérations mathématiques pour retrouver une distance sur le terrain à partir des données d'une carte, c'est apprendre qu'à une échelle donnée apparaissent tels ou tels phénomènes et qu'à une autre échelle, on découvre d'autres relations; c'est apprendre que le choix de l'échelle appropriée est une des opérations de base de tout raisonnement géographique et qu'il est dès lors capital de se poser les questions: à quelle échelle suis-je en train de penser? à quelle échelle l'auteur de mon document de référence se place-t-il? à quelle(s) échelle(s) dois-je aborder la problématique qui m'intéresse afin que je puisse en dégager des principes d'action?
Apprendre à penser les relations de l'homme à l'espace, c'est apprendre à utiliser sur le même modèle les concepts de localisation, d'espace produit, de maillage, de treillage, de hiérarchie et d'interaction, à poser les questions essentielles qui se dégagent de chacun de ces concepts, et à y répondre de manière de plus en plus complexe au fur et à mesure que l'apprentissage progresse. Certes, concevoir la géographie comme l'étude des relations de l'homme avec l'espace impose la construction de modèles, opération très abstraite. Mais, à l'école obligatoire, le jeune est là notamment pour apprendre à se construire des modèles abstraits, c'est une des opérations mentales des plus complexes, on le sait bien, et la géographie en offrant comme point de départ le moi, les autres autour de moi et l'espace dans lequel nous évoluons tous, permet, à notre sens, une construction plus contextualisée des indispensables modèles abstraits. A partir de l'espace le plus proche, à partir de l'utilisation quotidienne par les élèves de cet espace, la géographie scolaire peut permettre aux jeunes d'apprendre à se décentrer, à constater que l'autre n'a pas forcément la même utilisation de cet espace, qu'il n'en a pas la même représentation et que tout espace n'est donc pas simplement un donné, qui relèverait d'une vérité absolue, mais qu'il est vécu, qu'il est perçu et qu'à travers tous ces vécus et ces perçus, il est finalement construit. Dès lors, il devient légitime de se poser la question «qui construit l'espace et pour quel intérêt?», question qui ne peut avoir que deux types de réponses: ou on laisse le plus fort imposer sa solution ou toute construction impose la participation active de tous, ce qui implique la maitrise de concepts et de notions permettant la prise en compte du vécu de l'autre, de ses représentations et, en amont, tout cela implique l'écoute de l'autre, l'apprentissage de la rencontre et de la communication dans le refus de la violence. Et nous voilà déjà revenus au cœur du projet éducatif cherchant à développer l'«humain», dans ce cœur où devraient se rejoindre toutes les disciplines scolaires dignes d'être retenues.

\section{Ouvrages cités}

JACQUARD, A. (1991): Voici le temps du monde fini. Ed. du Seuil, Paris.

MEIRIEU, Ph. (1993): L'envers du tableau. ESF éditeur, Paris. MORIN, E., et KERN, A. B. (1993): Terre-Patrie. Ed. du Seuil, Paris.

RAFFESTIN, C., et TURCO, A. (1991): Epistémologie de la géographie humaine. In: Bailly, A., et al. Les concepts de la géographie humaine. Masson, Paris.

UNESCO (1994): Un sentiment d'appartenance. Paris. 